Available online at http://bajas.edu.iq

https://doi.org/10.37077/25200860.2021.34.sp1.3

College of Agriculture, University of Basrah

\section{Basrah Journal of Agricultural Sciences}

E-ISSN: 2520-0860

\title{
Adoption of Precision Agriculture by Detecting and Spraying Herbicide
} using UAV

\author{
Samarth S. Prabhu ${ }^{1}$, A .Vishal Kumar ${ }^{3}$, Rajkumar Murugesan ${ }^{2}$, Jayit Saha ${ }^{1} \&$ Ishita \\ Dasgupta $^{1}$
}
${ }^{1}$ School of Computer Science and Engineering (SCOPE), Vellore Institute of Technology, India
${ }^{2}$ VIT Business School (VITBS), Vellore Institute of Technology, India
${ }^{3}$ School of Mechanical Engineering (SMEC), Vellore Institute of Technology, India
*Corresponding author E-mail: samarthprabhu.s2001@gmail.com
Received 31 March 2020; Accepted 29 February 2021; Available online 19 August 2021

\begin{abstract}
Farmers are facing the VUCA environment (volatile, uncertain, complex and ambiguous) and data indicating the contribution of farming to India's GDP has come down from $52 \%$ to $18 \%$ between 1951 and 2018, which is alarming. At this juncture, developing countries like India, where over $70 \%$ of the rural people depend upon the agriculture fields, adoption of disruptive technology (creative destruction) becomes the need of the hour, to enhance the crop yield and quality. Weeds are one of the major issues which severely affect the crop output. Unmanned Aerial Vehicle (UAV) or drone is recommended, to address the problem. Globally, the market for agriculture drones to move from $\$ 1.3$ billion to $\$ 6.52$ billion by 2026 . Globally agriculture is the second largest industry after construction in terms of drone adoption. But Indian farmers have difficulty in adopting (or) procuring UAV's, as the size of their farm is small, income is very less. Other problems associated with the adoption of UAV include knowledge transfer and training to farmers, service support and maintenance cost. DaaS (Drone as a service) model is proposed, for rural areas. This paper aims to focus on weed management by providing a safer and cost-effective solution. By integrating technologies like visible light (VIS), near-infrared (NIR) light on an Unmanned Ariel Vehicle along with a precise sprayer and a weed detection system backed up by a lithium-ion battery (for longer flight duration), can help the process of spraying weedicide efficiently. The accuracy of the tested model is $92.6 \%$ for far away detection module and 95.4 for close range detection. UAV's with sprayer protects the farmer and consumers from odour and side effects.
\end{abstract}

Keywords: Drones, KNN, OpenCV, Agriculture 5.0, United Nations Sustainable Development.

\section{Introduction}

Weeds carry a negative connotation, even though it brings minerals from subsoil to topsoil (Daphne Ewing - Chow, 2020). If it is not managed properly, weed can reduce the crop yield to $22 \%$ of the normal quantity (Bob Reiter, 2019). Weeds can reduce the value of 


\section{Prabhu et al. / Basrah J. Agric. Sci., 34(Special Issue 1): 21-33, 2021}

the field, add the cost of cleaning, increase the irrigation need and can hamper the crop yield. Crop yield does not match with the requirements of burgeoning population set (to grow from 7.6 bn today to 8.6 bn by 2030,10 bn by 2050 (Murugesan, et al., 2018) as mentioned by KPMG (Klynveld Peat Marwick Goerdeler) in Agri Disruption).

Farmers use chemicals called pesticides in the agriculture field ie...herbicides (Ram Swaroop et al., 2001) or commonly called as weed killers to manage weeds, fungicides to manage fungal disease and insecticides to manage bugs. Most traditional sprayers administer agrochemicals evenly, though the spread of weeds is normally patchy, resulting in wastage of important composition, enhanced expenses, crop devastation risk, pest resistant to hazardous chemicals resulting in environmental deterioration, adulteration of products and many more (Partel et al., 2019). Traditional method of weed removal by labour is time consuming i.e. creative destruction (Schumpeter, 1942) strategy is suggested to farmers. So, new solutions are required, to handle the current situation. Blue Ocean Strategy (Refer figure 1 - BoS - W.Chan Kim, Rene Mau Creative Destruction borgne, 2004) i.e. Usage of drones (or) Unmanned aerial vehicle (or) Autonomous aerial vehicle, has been adopted by farmers.

Experts have predicted that Agriculture to enjoy the maximum benefit by using Unmanned Aerial (UAV) vehicles (Lan \& Chen, 2018) or drones and $80 \%$ of domestic sale of drones in the USA [6], will be used by farmers, to increase the crop yield. Israel has used modern technologies and exported agriculture products worth USD 1.3 billion annually (Murugesan, et al., 2018). Use of
UAV's (Meng et al., 2020) in line with UN General Assembly's sustainable development objectives for the year 2030 (Rosenthal, 2018) which includes doubling productivity of agriculture (Murugesan, \& Sudarsanam, 2019). This paper focuses on reducing these negative impacts by integrating the emerging technologies like open $\mathrm{CV}$ and Gaussian filter which distinguishes between target weeds (Torres-Sánchez, et al., 2013) and other nontarget plants through ground control points (GCP) (Gómez-Candón et al., 2014) and then removes them by spraying weedicide in the target location. This paper refers to Agriculture 5.0 (Murugesan, et al., 2019). Use of Artificial Intelligence and Unmanned vehicles in agriculture). This project comes under precision agriculture, (Torres-Sánchez et al., 2013) a rapidly growing area where high accuracy and controlled environment makes the work of human easier reducing the wastage of yield.

DisTA" (Disruptive Technologies for Agriculture) include Reinforcement learning (Sutton \& Barto, 1998), Swarm Robotics, (Carbone et al., 2018) Cyber Physical Systems, Drones, Fog (or) Edge computing have to be used, for effective monitoring of weeds.

To provide innovative, cost effective solution using disruptive technologies and to solve this social problem (weed removal) Academia, Corporate, NGO's, Microfinance companies, Agriculture Experts, Government, Domain experts can join together (Trans disciplinary approach). 


\section{Materials \& Methods}

\section{For drone}

1. BLDC (brushless DC motor): EMAX MT2213 935KV 2212 BRUSHLESS MOTORS (Kale et al., 2015).

In terms of thrust, this set is capable of providing a value of 850 grams per volt. Here BLDC motor is because the speed is adjusted according to the input voltage which is useful in avoiding collision which may include moving up or down.

2. ESC: The ESC (Electric Speed Controller) generates three high frequency signals with adjustable phases continually to keep the motor turning. The ESC provides current to the motor according to height of flight or amount of herbicide to be sprayed. The ESC is a controller board that gives out a three-phase output for battery input. The frequency of the signals vary, but for a quadcopter it is better if it can respond to higher frequency as speed of the motor can be adjusted quicker for optimal functioning which sometimes may be very crucial to avoid collisions.

3. DC: 30PRM 12 v DC geared motors known for their application in robotics and related field. These can be used as they are small in size, can be replaced easily if a more force is needed and are also efficient.

4. Accelerometer Sensor: An accelerometer is a device used to measure acceleration forces like gravity. Three axes accelerometer help us orient the drone in all the three coordinates (X axis, $\mathrm{Y}$ axis and $\mathrm{Z}$ axis). Acceleration is calculated as change in velocity per unit time.
- $\pm 2 \mathrm{~g}, \pm 4 \mathrm{~g}, \pm 8 \mathrm{~g}$ and $\pm 16 \mathrm{~g}$ accelerometers are useful in the perfect calibration, which ideally operates at $500 \mu \mathrm{A}$

- $10 \mu \mathrm{A}$ at $1.25 \mathrm{~Hz}, 110 \mu \mathrm{A}$ at $40 \mathrm{~Hz}$ for lower power accelerometer mode

- Customer - programmable interrupts and self-tests

5. Gyroscope Sensor: A gyroscope measure angular velocity, it is a disk kind of structure that is mounted which is free to move in along any axis helping in maintaining the stability of the drone

A user programmable angular rate sensor with full scale(X, Y, $\mathrm{Z}$ axis) gyroscope of range from \pm 250 to $\pm 2000 \% \mathrm{sec}$

- External sync facilitates image, video and GPS synchronization

- Integrated 16-bit ADCs enable simultaneous sampling of gyros

- Digitally-programmable low-pass filter

- Standard operating current: $3.6 \mathrm{~mA}$ with standby current usage to be $5 \mu \mathrm{A}$

\section{Li-Po battery}

This is the commonly used li-po batteries. As an alternative LiHV (lithium polymer high voltage) can also be used

7. IoT (Internet of Things): for showing real time images of their crops, weeds in the field and also to redirect the drone to places where it might have missed due to various factors.

8. GPS: GPS (global positioning system) will also be used to restrict the drone to a particular boundary, generally to an individual's field or a group of individuals and also to direct the drone back to its starting point. 


\section{Prabhu et al. / Basrah J. Agric. Sci., 34(Special Issue 1): 21-33, 2021}

Integration of all this would ensure that the drone avoids the obstacles which may damage the drone and also they ensure ease of use in hilly areas where elevation will either increase or decrease while spraying

9. Camera: A high resolution camera is equipped with a wide spectrum of color capturing but not so wide degree of viewing as more viewing angle especially beyond $120^{\circ}$ (chances of misinterpreting) which is further processed to find the desirable and undesirable plants.

\section{Software}

\section{Arduino}

Arduino is a tool for making PC's which can feel and regulate more of the environment than your PC. It's an open source physical computing platform based on a micro controller board with an unique environment for writing code. Arduino can be used to manage the parts and also to process the input and proceed with a result. The Arduino programming language is an implementation of wiring (a physical computing platform), which is based on processing multimedia programming environment

\section{Open CV}

Open CV is the most advanced computer vision and machine learning library. This library has around 2000+ algorithms for various activities including recognize frontispiece, identify widgets, label human behaviour in videos, trace camera journey, track moving article, extract 3D models of articles, produce 3D point clouds from cameras, combine images to produce a high resolution image of an entire scenario, distinguish similar images from an image index delete red eyes from photos taken using light, chase eye movements, identify sceneries and etc. It supports programming languages like $\mathrm{C}++$, Python which are used along with pandas and NumPy, Java etc. The computer reads any image as a matrix also known as pixels where each one is a specific combination of the primary colors red, blue and green.

Our project identifies the clusters where weed growth is present and generates a percentage to indicate that. A higher percentage indicates greater growth of weed in that area. Gaussian filter successfully divided the image area based on weed growth percentage. Moreover the methodology focuses on taking both images as well as video as a viable input, thus improving the application by a manifold. This methodology takes a huge dataset of Weed classified pictures, which is then passed into the neural network, and the parameters are set accordingly to classify the image as weed prone or not.

\section{Expectation maximization algorithm}

Expectation maximization algorithm can be used for latent variables (meaning the variables that are there because of the inference of some other variable as these cannot be observed actually) too to predict the value according to the given conditions

\section{Algorithm}

Expectation step (E-step): Using the given specification and the previously observed data, the missing values are estimated

Maximization step (M - Step): Here, values generated in E - step is evaluated, to check if any other specification can be exercised and is amended, if something is found. 
Prabhu et al. / Basrah J. Agric. Sci., 34(Special Issue 1): 21-33, 2021

The above two steps are repeated until we get a much clear data or in other words till it reaches the convergence point.

The biggest advantage we have in implementing this algorithm is that every time the iteration continues the likelihood of detection increases as new and new parameters will be included and also in most cases the implementation of $\mathrm{E}$ and $\mathrm{M}$ steps is pretty easy

\section{KNN Algorithm:}

K-nearest neighbours (KNN) algorithm can be used for crop yield prediction (CYP).

Properties that define KNN:-

Lazy learning algorithm - KNN is a lazy learning algorithm because it does not have a specific dataset from where it trains itself in turn it uses all the data it has while classification.

Non-parametric learning algorithm - KNN is also a non-parametric learning algorithm because it doesn't assume anything about the implicit data.

It classifies a data point based on how its neighbors or input data is classified. KNN classifies new cases based on similarity with its nearest neighbor. $\mathrm{K}$ refers to the number of nearest neighbors to include into the comparison for identification. What basically happens is it calculates distance of itself with every point on the dataset and based on the $\mathrm{k}$ value selects $\mathrm{k}$ distances in ascending order and compares.

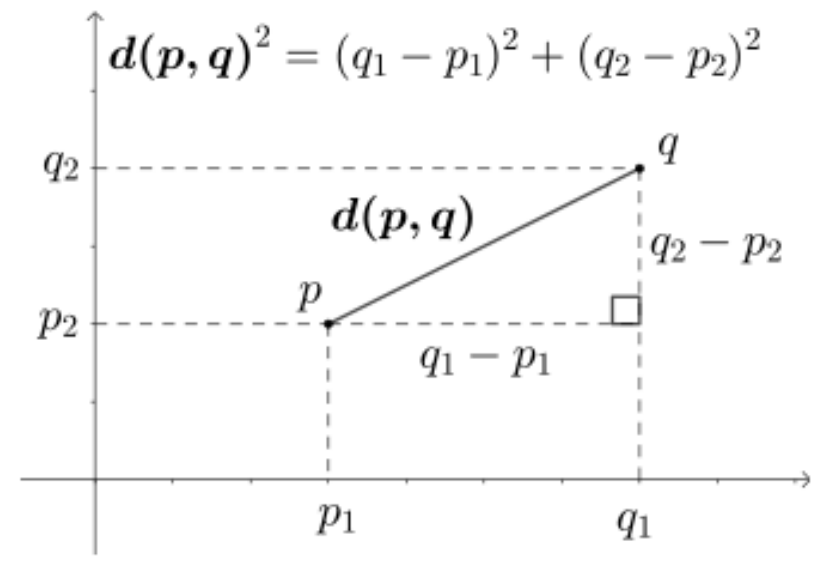

Fig. (1): Eucleidean distance formula.

For example if $\mathrm{k}$ is 4 it takes the lowest 4 distances and sees how they are labelled and gives output accordingly.

Higher the value of $\mathrm{k}$ slower it would be because it would require a lot more comparison and also even is not preferred as it might lead to a tie situation while comparing. Usually $\mathrm{k}$ is taken to be the square root of the number of values.
Image description:

\section{Fig. (1): Euclidean distance}

The formula above mentioned which helps us calculate the distance between any two points on a plane or a $3 \mathrm{D}$ fig. using the two and three co-ordinates respectively which uniquely represent the point. We find the horizontal and vertical distance between them which make the sides of an imaginary right triangle and 


\section{Prabhu et al. / Basrah J. Agric. Sci., 34(Special Issue 1): 21-33, 2021}

hypoteneous becomes the distance and we use pythogoreas theorem to find that length.

\section{Neural Network in Weed Detection (Fig. 2)}

A feature map of $19 \times 19 \times 256$ was created after the convolutional layers. The resultant image was passed into a Inception V3 network layer. It resulted in a $19 \times 19 \times 21$ resolution, to convert the 2-dimensional tensor to 3dimensional tensor. The YOLO v3 algorithm is used to create the bounding boxes, which were encoded to surround on weed-laden areas via other algorithms and annotation processes. Also, they have the accuracy with which it predicted the possibility of a weed infestation. Various Machine Learning algorithms were tested to predict the bounding boxes, and it was observed that K-NN algorithm gave the highest accuracy. The following figure shows 24 convolution layers which have 2 dense or fully connected layers following them. Reduction layers have been used to lessen the feature map depth. The tensor of shape $(7,7$, and 1024) is output of the last layer. Then, flattening of tensor followed by regression resulted in $7 \times 7 \times 30$ parameters $(7,7$, and 30$)$ boundary box for each prediction location.

In fig. (3) the importance of weed detection and weed prevention has been highlighted to give a clear picture of the present demands. The four methods of reducing labor dependency, eliminating wastage, raising crop yield and creating good quality yield are the golden rules of any successful agricultural practice performed, in our case weed detection.

Fig. (4) mainly classifies the region partitions into weed and non-weed. The segment of the frame having more quantity of weeds in proportion to other partitions are highlights with a score of higher percentage as compared to others. Clearly the crops having weeds around them is identified in the middle segment of the image having a percentage score of 35.99 while others have only less than or around 10 .

Fig. (5) segment analysis done on captured frames has been shown in this figure. Image segmentation into more simpler and specific requirements is an important part of image classification which has been done on captured image of weeds in this particular figure. Segmentation done in groups have gradually reached the final layer of accurate identification of the croplands as green vertical lines, a result of Deep Learning Image Segmentation on captured images.

Fig. (6) an optimization algorithm, the function that is being used primarily a neural network, to evaluate a candidate solution (i.e. a uniform/non-uniform set of weights) is referred to as the objective function. We achieve to maximize or minimize the objective function, meaning that we are searching for a candidate solution that has the highest or lowest score respectively. It is very imperative to minimize the loss, and accuracy is thus always accompanied with it as a metric to predict the nature of the algorithm. As visually evident, the loss decreases with every epoch, just the model is not over fitting and is in tandem with the accuracy.

Fig. (7) represents the training and validation set testing accuracy based on hyper parameterized CNN Algorithm

\section{Result \& Discussion}

A state of the art weed detection mechanism has been finely developed (Figs. 2, 5 and 6). In this model, Open $\mathrm{CV}$ is used to its entire 
Prabhu et al. / Basrah J. Agric. Sci., 34(Special Issue 1): 21-33, 2021

potential, as it enables to detect the weed growing from drone range as well as from a hand wide distance. The accuracy of the tested model is $92.6 \%$ for far away detection module and 95.4 for close range detection.

$$
\begin{aligned}
& \lambda_{\text {coord }} \sum_{i=0}^{S^{2}} \sum_{j=0}^{B} \prod_{i j}^{o b j}\left[\left(x_{i}-\widehat{x}_{l}\right)^{2}+\left(y_{i}-\widehat{y}_{l}\right)^{2}\right]+\lambda_{\text {coord }} \sum_{i=0}^{S^{2}} \sum_{j=0}^{B} 1_{i j}^{o b j}\left[\left(\sqrt{w_{i}}-\sqrt{\widehat{w}_{i}}\right)^{2}+\left(\sqrt{h_{i}}-\sqrt{\widehat{h_{j}}}\right)^{2}\right] \\
& +\sum_{i=0}^{S^{2}} \sum_{j=0}^{B} 1_{i j}^{o b j}\left(c_{i}-\widehat{c}_{l}\right)^{2}+\lambda_{\text {noobj }} \sum_{i=0}^{S^{2}} \sum_{j=0}^{B} \prod_{i j}^{\text {noobj }}\left(c_{i}-\widehat{c}_{l}\right)^{2}+\sum_{i=0}^{s^{2}} 1_{i}^{o b j} \sum_{c \in \text { classes }}\left(p_{i}(c)-\widehat{p}_{l}(c)\right)^{2}
\end{aligned}
$$

Where the first two terms are localization next two are confidence and last one classification. The feed picture is first taken and then bitwise operations are performed on it,
Accuracy formula:

Loss Function making a mask of the model. This mask is prepared on the basis of the recognition with the neural fed data.

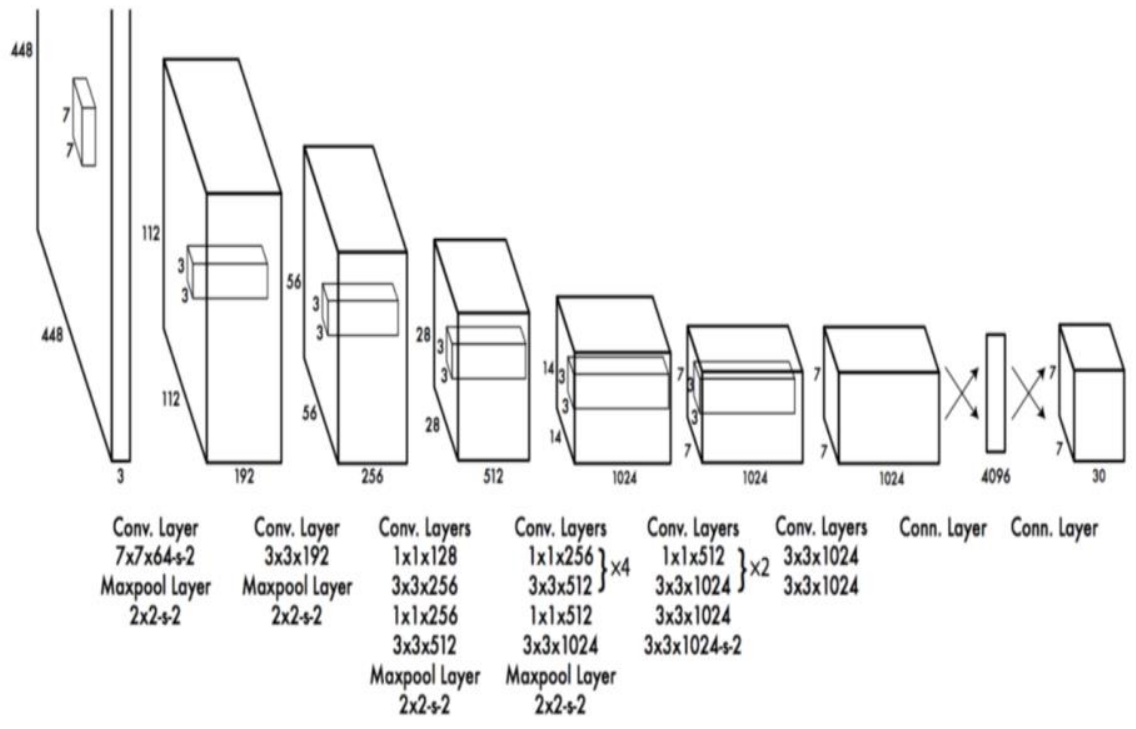

Fig. (2): Neural network design.

In the illustrations attached, there are weed inflicted area in the field in the form of percentage. This model will be immensely helpful as it will designate high weed pestered regions and thus helping the farmers to clear huge hectares of fields. This will have a huge impact on the agricultural sector and thus improving the crop yield significantly. 
Prabhu et al. / Basrah J. Agric. Sci., 34(Special Issue 1): 21-33, 2021

The data analysis was primarily executed ny plotting the accuracy metrics and the loss metrics with each increasing epoch. The main goal was to maximize the tradeoff between the Thus we visualized the loss function to decrease with every epoch and the accuracy to improve. Moreover, the image segmentation was performed on the weed images, so that the implemented algorithm, could paralelly divide the analysis in its own frame of reference. Thus this methodology, increased the performance of the model. Initially the whole picture was passed into the neural network. But after refining, the model was trained on segments of a full picture, and each segment of a picture was marked sequentially, to keep track. This normalization resulted in greater real time processing speed, giving high accuracy and efficiency on real time analysis.

Also the analysis of the data analysis algorithms have been provided to support the accuracy report and also the training vs testing graph. Thus, it is the need of the hour to accuracy and loss, so as to ensure the model fitted well. A too high accuracy on test data generally indicates an overfitting model. This type of model in practicality performs badly. eliminate weeds because they tend to deter the growth of the healthy and useful crop.

\section{Challenges}

Following are the few challenges which this technology might face

\section{Investment}

Lack of funds to invest in these kinds of technology and especially in developing countries where agriculture is mostly done in rural areas mostly by the economically weaker sections. Another major issue is the size of the field they own, unlike developed countries agriculture in lesser developed nations is done in small scales where people have a small piece of land which further gets smaller for the future generations making their income from agriculture marginal. This creates hesitation in investing in newer technology.

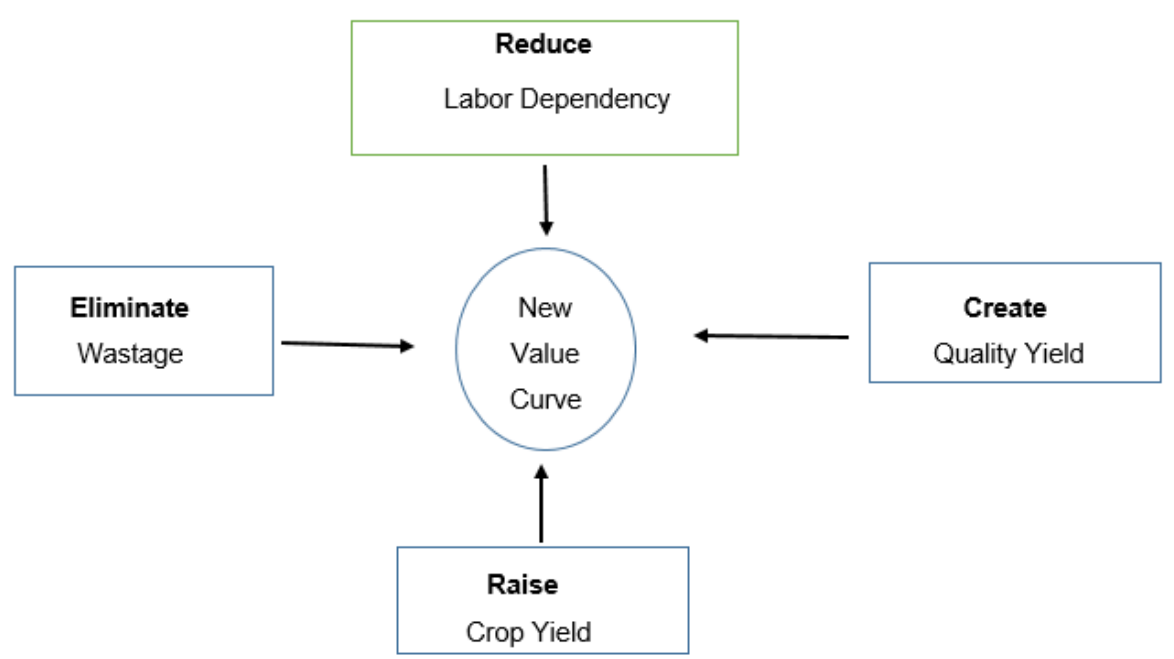

Fig. (3): Blue Ocean strategy. 
Prabhu et al. / Basrah J. Agric. Sci., 34(Special Issue 1): 21-33, 2021

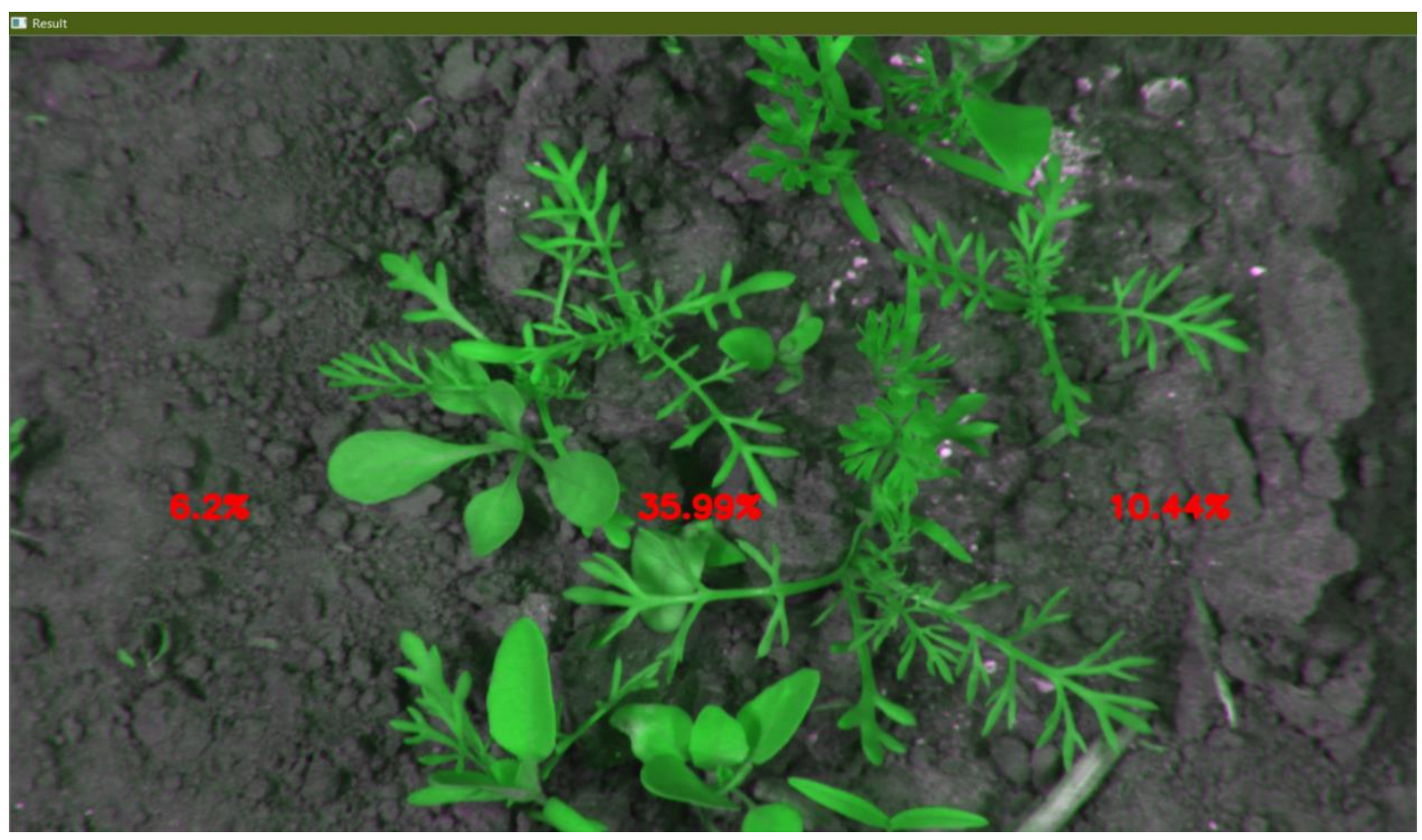

Fig. (4): Close view.

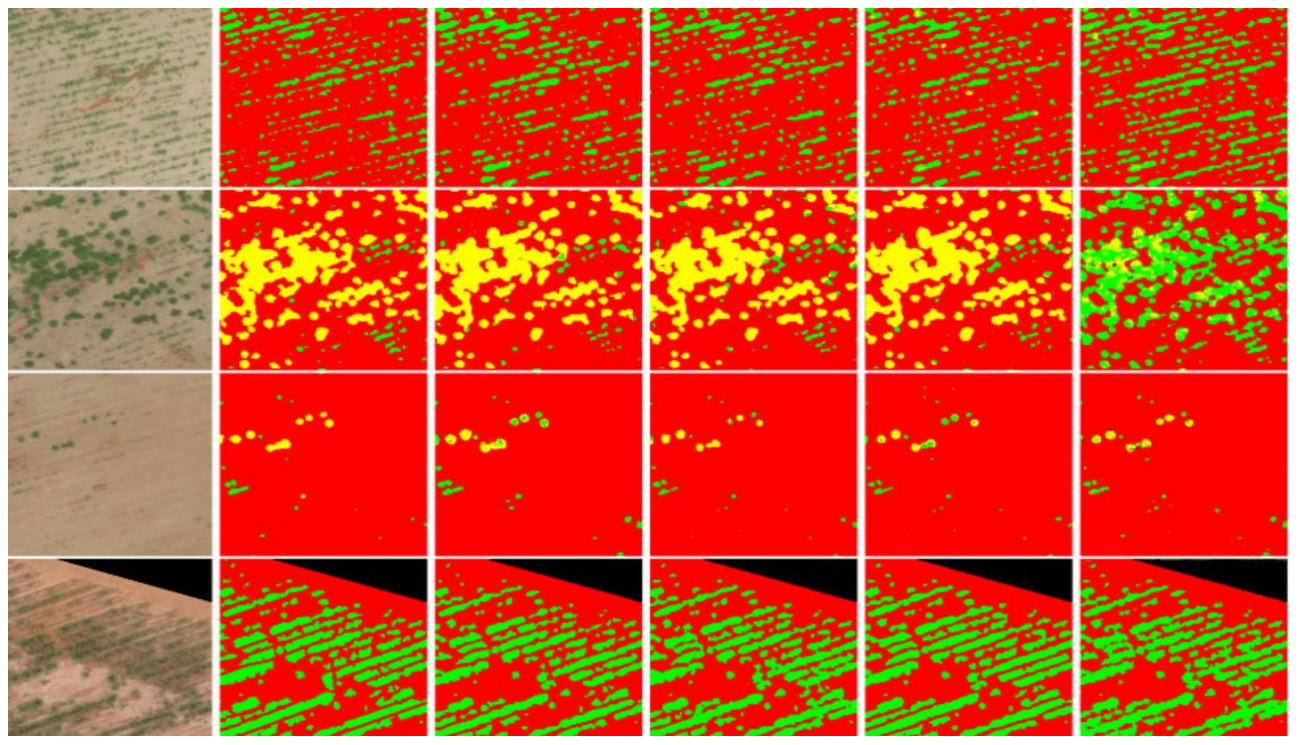

Fig. (5): Segment analysis of crop field.

\footnotetext{
The $5^{\text {th }}$ International Conference on Agricultural and Food Engineering (CAFEi) 2021
} 
Prabhu et al. / Basrah J. Agric. Sci., 34(Special Issue 1): 21-33, 2021

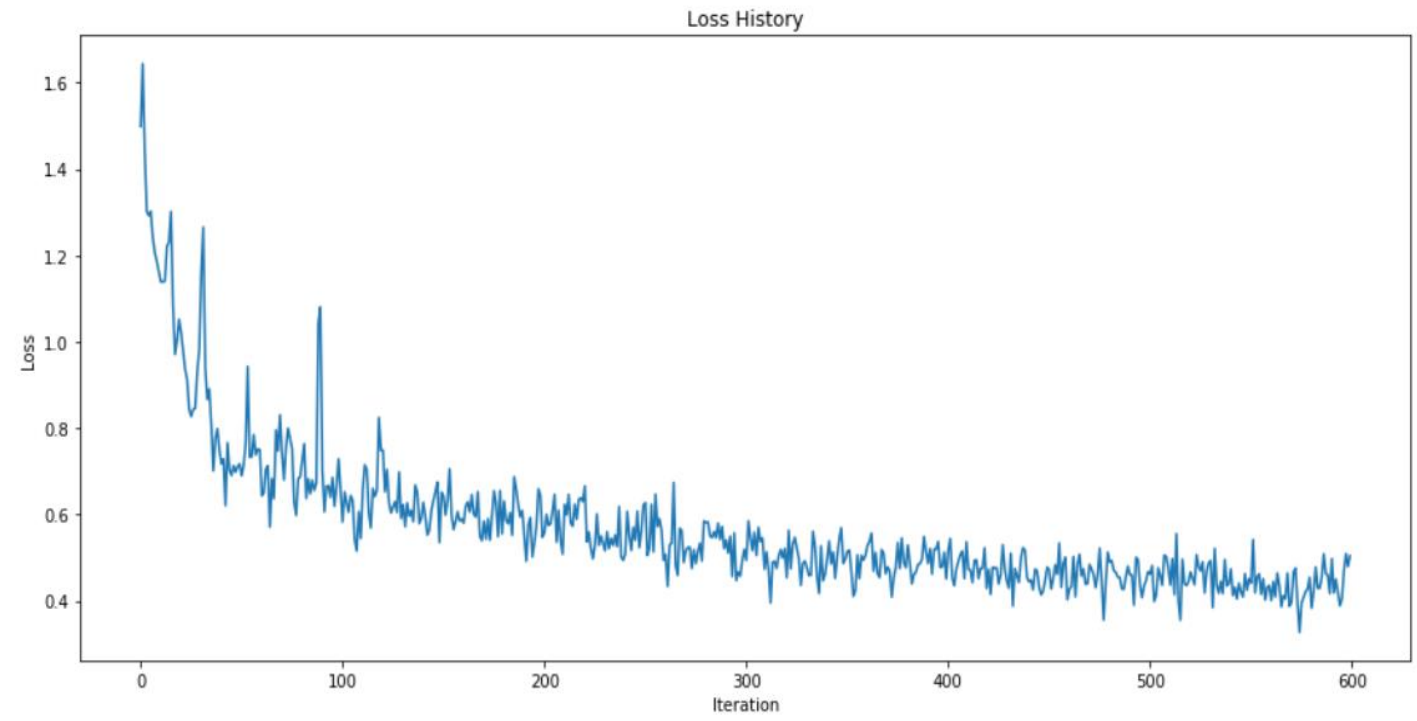

Fig. (6): Loss history analysis.

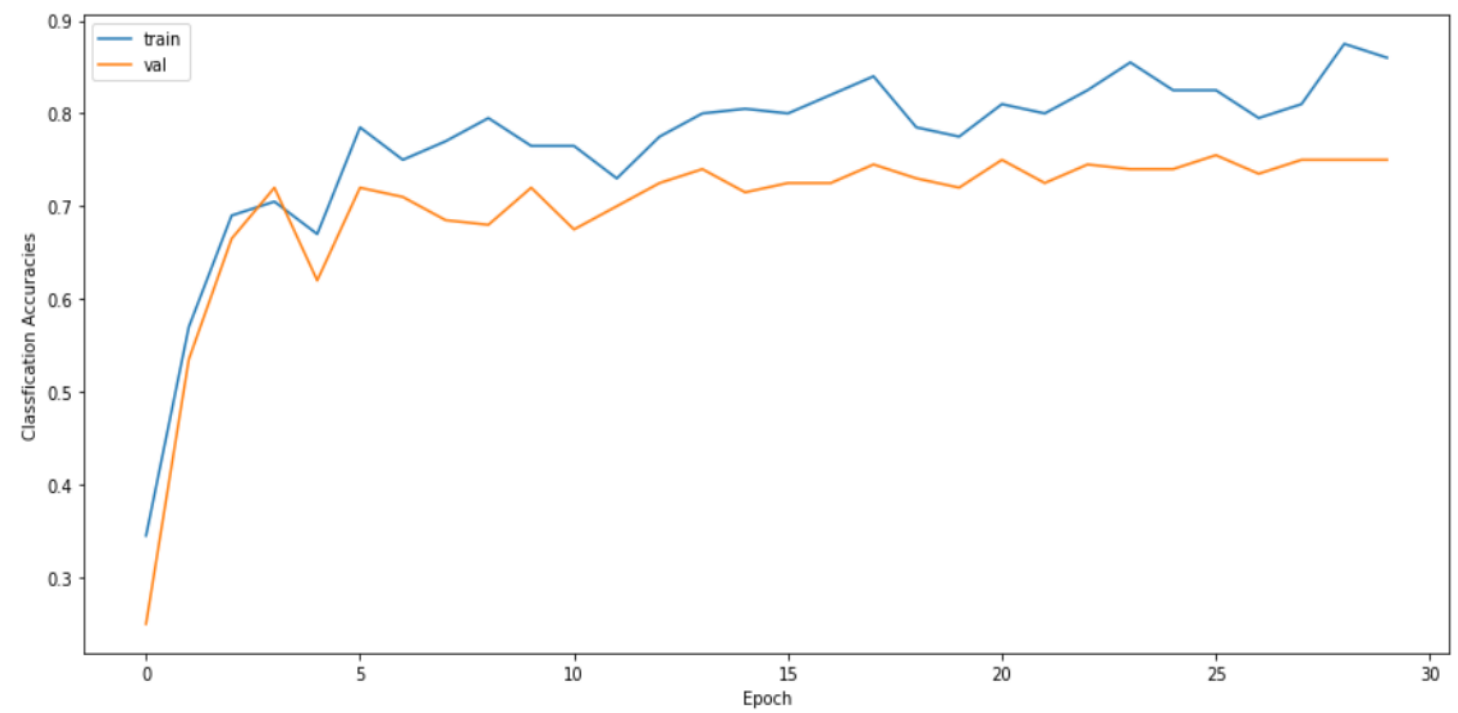

Fig. (7): Classification accuracies.

In countries like India where generally the eldest of the family decides on what to do the suggestions of the younger generation is not considered much, in other words, meaning there is a barrier kind of setting which is blocking people from trying new techniques

\section{Climatic conditions}

Adoption of such technology also depends upon the climatic conditions as the drone will not be able to fly in windy places and even if it does the spraying level should be quite close to the weed else there is a chance of weedicide to get sprayed over the wrong plants. Drone with AI and GPS data, can ensure that spraying is done at the right place only. The amount of 


\section{Prabhu et al. / Basrah J. Agric. Sci., 34(Special Issue 1): 21-33, 2021}

downpour also matters as the drone may fly in light rain but may not be able to in heavy rain and also rain might wash the weedicide off the weed which also cannot be avoided even if sprayed over the full field.

As a remedy to this, it is best if we identify weeds in the early stage of crop growth where there will not be much difference between crop height and the weed height making it easier to identify and spray as it would be close to the ground level.

Due to environmental conditions, Inspection of farm by Quadcopter (or) Drones (or) UAV (Shakhatreh et al., 2019) has limitations like maximum flight speed, maximum flight height and battery time (Albani, et al., 2017). Swarm Robot (Tan \& Zheng, 2013) with sensors like LIDAR, Thermal imaging camera, RGB Stereo camera, Multi spectral camera (Das et al., 2015) to get better resolution of field images, for analysis. Due to battery time of UAV, coverage is less per trip. Swarm of drones is efficient in addressing this challenge (Perez, 2020). Swarm Drones from Chinese company $\mathrm{XAg}$ is working in 35 countries now, for weed removal. The solution is based on a reinforced random walk with inhibition of return. Hyperspectral imagery (Murugesan \& Sudarsanam, 2020) has information measured in 150 narrower bands, each has the width of 5 nanometers. They can provide an incredible capacity to see that which cannot be seen by the human eye.

\section{Duration of flight}

Being a new and upcoming field the main problem faced by most of the drone industry is the duration of flight (around 20 minutes) because when it comes to these kinds of large scale applications battery life also plays a major role. If we try to increase the battery size the weight of the drone would increase needing more thrust for it to lift from the ground. Other options available in other countries includ... i) Hydrogen fuel cell drones that can fly for 1 hour, ii) Petrol electric hybrid drone, which can fly for 2 hours. These are not available in countries like India. Drone to fly at a height of 5 feet above the crop. Drone to cover 1 acre of field in 12 minutes. Exact location ( $\mathrm{x}, \mathrm{y}$ coordinates from one end) to be sprayed is available through GPS. Drone to move to exact location using this coordinates \& move to the next. Spraying the affected area (within 1 acre), will be done in 4 to 6 minutes max. Crop and weed is identified from the images taken by high pixel camera, fixed with drone. Data is collected between $11 \mathrm{am}$ and $2 \mathrm{pm}$, considering the availability of sun light to get best pictures.

\section{Training and support:}

Providing training and support may be another issue we must look through as accessibility might be less in rural areas. Training could be provided at the time of purchase but support remains a major challenge. In case of small tweaks needed can be done maybe through an online live tutorial or so but in case any part is not working because of wear and tear or maybe some human error, a replacement can be a bit difficult, they might have to go to the nearest city to get the parts. Academia near the village can extend support to the farmers, during weekends. Non-Government Organization can also step in, for this purpose. Indian Government has recommended every engineering institution, to adapt and support 2 villages.

\section{Sun angles and shadows}




\section{Prabhu et al. / Basrah J. Agric. Sci., 34(Special Issue 1): 21-33, 2021}

The solution is dependent on image recognition (Duffy et al., 2017), there is a chance that it might fail to detect because of the angle of the sun, the shadows formed

\section{DaaS}

Drone as a service model is recommended. Tractor is given on rental basis to the needy, in villages. This leads a win situation and so it has been successful in rural areas. Additional revenues for the owner and availability of the solution for the needy on rental basis). Similarly Drone can be offered by the owner to the needy. Unemployed youths of villages can also start earning, as drone operator.

\section{Conclusion}

Farmers suffer due to weed. We propose the usage of drones, to bring down the losses (due to weed growth) to near zero. Problems associated with acquisition of drones, training required to use, maintenance etc. has been addressed in our paper. Hybrid powered drone with AI and GPS Data, can save money for farmers. With Knowledge, Training, Maintenance support, DaaS (OPEX model preferred by farmers), financial support at competitive rates during crisis, Innovation and support from others will enable the adoption of Agriculture 5.0 easily. This will be in line with the recommendations of Late President of India Padma Shri. Dr. A.P.J. Abdul Kalaam (provide urban amenities in rural areas "PURA Scheme"). This can also lead to achievement of United Nations Sustainable Development (UNSD - 2030) goals and the vision on Indian Prime Minister Shri. Narendra Modi (i.e. to double the income of farmers). Physical efforts of farmers are reduced and identification of $100 \%$ weed is achieved through usage of drones. Objective of i) getting higher crop yield, ii) reducing the wastage/ loss and giving technology to illiterate farmers is achievable. This will result in a) enhancing income, b) improving purchase power and giving better standard of living to Indian farmers.

\section{Conflicts of interest}

The authors declare that they have no conflict of interests.

\section{References}

Albani, D., Nardi, D., \& Trianni, V. (2017). Field coverage and weed mapping by UAV swarms. IEEE. https://ieeexplore.ieee.org/document/820629/aut hors

Ram Swaroop Meena, Sandeep Kumar, Rahul Datta, Rattan Lal, et al., (2020), Impact of Agrochemicals on Soil Microbiota and Management: A Review .MDPI, Land

Bob Reiter, R. (2019). The crop protection toolbox: How farmers defend their crops from monster weeds Forbes.https://www.forbes.com/sites/bayer/2019/11/ 07/the-crop-protection-toolbox-how-farmers-defendtheir-crops-from-monster-weeds/?sh=27151fc $81 \mathrm{f} 28$

Carbone, C., Garibaldi, O., \& Kurt, Z. (2018). Swarm robotics as a solution to crops inspection for precision agriculture", ESTEC Conference Proceedings 6th Engineering, Science and Technology Conference. 2018. 552-562. file://C:/Users/hassan /Downloads /1459-Article\%20Text-7799-1-10-20180211.pdf

Daphne Ewing - Chow, (2020). Wild Waste: It's time to rethink the war on weeds, Forbes. https://www.pinterest.nz/pin/446419381816740168/? amp_client_id=CLIENT_ID(_)\&mweb_unauth_id= $\% 7 \mathrm{~B} \%$ 7Bdefault.session\%7D\%7D\&amp_url=https\% $3 \mathrm{~A} \% 2 \mathrm{~F} \% 2 \mathrm{Fwww}$.pinterest

Duffy, J. P., Cunliffe A. M., \& De Bell, L. (2017). Location location location considerations when using lightweight drones in challenging environments, Remote Sensing in Ecology and Conservation, 113.https://doi.org/10.17863/CAM.13118

Gómez-Candón, D. ,De Castro A.I., López-Granados F. (2014). Assessing the accuracy of mosaics from unmanned aerial vehicle (UAV) imagery for precision agriculture purposes in wheat", IEEE 


\section{Prabhu et al. / Basrah J. Agric. Sci., 34(Special Issue 1): 21-33, 2021}

Conference on Computer Vision and Pattern

Recognition. Precision Agriculture, 15, 44-56. https://doi.org/10.1007/s11119-013-9335-4

Kale, S. D., Khandagale , S. V., Gaikwad, S. S., Narve, S. S., \& Gangal, P. V. (2015). Agriculture Drone for Spraying Fertilizer and Pesticides International Journal of Advanced Research in Computer Science and Software Engineering, 5, 804-807. https://www. scribd.com/document/417477409/Agriculture-Dronefor-Spraying-Fertilizer-and-Pesticides

Lan, Y., \& Chen, Sh. (2018) Current status and trends of plant protection UAV and its spraying technology in China", International Journal of Precision Agricultural Aviation, 1, 1-9. https://pdfs. Semantic scholar.org/b385/f511727b5cd67c2f5069199842c36e 4a91ed.pdf

Meng, Y., Su, J., Song, J., Chen, W., \& Lan, Y. (2020). Experimental evaluation of UAV spraying for peach trees of different shapes: Effects of operational parameters on droplet distribution, Computers and Electronics in Agriculture 170, https://doi. Org /10. 1016 /j.compag.2020.105282

Murugesan, R., \& Sudarsanam, S. K. (2019). Transdisciplinary Approach for Sustainable Rural Development, IJRTE, 8, 2453-2460 https://www. ijrte.org/wp-content/ uploads /papers /v8i1 /A222905 8119.pdf

Murugesan, R., \& Sudarsanam, S. K. (2020). Development of smarty farming framework. Test Engineering and Management, 83, 8474 - 8484.

Murugesan, R., Sudarsanam, S. K., \& Shanmugasundaram, S. (2018). Industry 4.0 for sustainable development. Annual Technical Volume of the Institution of Engineers, 3.

Murugesan, R., Sudarsanam, S. K., Malathi. G., \& Varadarajan, V. Venkataraman, N., Venugopal, R., Rekha, D., Saha, S., Bajaj, R., Miral, A., \&
Venkataraghavan, M. (2019). Artificial Intelligence and Agriculture 5.0, IJRTE

Partel, V., Kakarla, S. Ch., \& Ampatzidis, Y. (2019) Development and evaluation of a low - cost and smart technology for precision weed management utilizing artificial intelligence Computers and Electronics in Agriculture, 157, 339-350. https://doi.org/10.1016/j.compag.2018.12.048

Perez, G. (2020). Weed spotting by drone, UC Weed Science. https://ucanr.edu/blogs/blogcore/post detail .cfm?postnum $=25759$

Rosenthal, A. (2018). Drones for development: Ho UAV's are supporting the global goals, UN Foundation, https://unfoundation.org/blog/post/dro nes-for-development-how-uavs-are-supporting-theglobal-goals/

Schumpeter, J. A (1942). Capitalism, Socialism and democracy. Harper \& Brothers, 431pp.

Shakhatreh, H., Sawalmeh, A., Al-Fuqaha, A., Dou, Z., Almaita, E., Khalil, I., Othman, N. Sh., Khreishah, A., \& Guizani, M. (2019). Unmanned Aerial Vehicles (UAVs): A survey on civil applications and key research challenge. In IEEE Access, vol. 7, pp. 48572-48634. doi: 10.1109/ACCESS.2019.2909530.

Sutton, R. S., \& Barto, A. (1998), Reinforcement learning, MIT Press, 344pp. https://www.Andrew .cmu.edu/course/10-703/textbook/B arto Sut ton .pdf

Tan, Y., \& Zheng, Z. Y. (2013). Research Advance in Swarm Robotics, Defense Technology, 9, 18-39, https://doi.org/10.1016/j.dt.2013.03.001

Torres-Sánchez, J., López-Granados, F., De Castro, A. I., \& Peña-Barragán, J. M. (2013). Configuration and specifications of an Unmanned Aerial Vehicle (UAV) for early site specific weed management. PloS one, 8 , e58210. https://doi.Org/10.1371/journal.pone.0058 210 